\title{
Model sensitivity and uncertainty analysis using roadside air quality measurements
}

\author{
Sotiris Vardoulakis ${ }^{1,3}$, Bernard E.A. Fisher ${ }^{2, *}$, Norbert Gonzalez-Flesca ${ }^{1}$ \& Koulis Pericleous ${ }^{3}$ \\ IInstitut National de l'Environnement Industriel et des Risques (INERIS), Parc Technologique ALATA, BP 2, \\ 60550 Verneuil-en-Halatte, France \\ 'National Centre for Risk Analysis \& Options Appraisal, Environment Agency, Kings Meadow House \\ Kings Meadow Road, Reading RG1 8DG, UK \\ 'School of Computing and Mathematical Science, University of Greenwich, Maritime Greenwich \\ University Campus, 30 Park Row, London SE10 9LS, UK
}

\begin{abstract}
Most of the air quality modelling work has been so far oriented towards deterministic simulations of ambient pollutant concentrations. This traditional approach, which is based on the use of one selected model and one data set of discrete input values, does not reflect the uncertainties due to errors in model formulation and input data. Given the complexities of urban environments and the inherent limitations of mathematical modelling, it is unlikely that a single model based on routinely available meteorological and emission data will give satisfactory short-term predictions.

In this study, different methods involving the use of more than one dispersion model, in association with different emission simulation methodologies and meteorological data sets, were explored for predicting best $\mathrm{CO}$ and benzene estimates, and related confidence bounds. The different approaches were tested using experimental data obtained during intensive monitoring campaigns in busy street canyons in Paris, France. Three relative simple dispersion models (STREET, OSPM and AEOLIUS) that are likely to be used for regulatory purposes were selected for this application. A sensitivity analysis was conducted in order to identify internal model parameters that might significantly affect results. Finally, a probabilistic methodology for assessing urban air quality was proposed.
\end{abstract}

Lelwords: Air pollution; Model sensitivity; Uncertainty; Street canyon; Traffic emissions; Meteorological data

*Corresponding author: B.E.A. Fisher

E-mail: bernard.fisher@environment-agency.gov.uk, Tel: +44 (0) 1189 535244, Fax: +44 (0) 1189535265

\section{Introduction}

Mathematical modelling has been widely used for assessing ambient air quality. Government departments, agencies, and local authorities increasingly (but not exclusively) rely on air pollution models for making decisions related to air quality and traffic management, urban planning, and public health. As a result, the model users' community is becoming larger and more diverse.

Most of the air quality modelling work has been so far based on the "deterministic" approach of using only one dispersion model for a specific application. The selected model provides estimates of average concentrations using a specific meteorological and emission data set. A serious weakness of this method lies on the fact that many uncertainties, not only related to the calculations and input variables, but also to the very nature of atmospheric processes, are ignored. That might have serious implications for exposure studies, since the area and number of people exposed to a predicted pollution level may be very sensitive to the uncertainties associated with this prediction (Fisher and Ireland, 2001).

The total uncertainty involved in modelling simulations can be considered as the sum of three components (Hanna, 1988): (a) The uncertainty due to errors in the model physics, (b) the uncertainty due to input data errors, (c) the uncertainty due to stochastic processes (e.g. turbulence) in the atmosphere. It may be possible to reduce the first component of model uncertainty by introducing more physically realistic and computationally efficient algorithms. It may also be possible to eliminate some of the effects of input data errors once more accurate monitoring instruments can be set up at representative locations. However, the stochastic fluctuations are a natural characteristic of the atmosphere that cannot be eliminated. Therefore, practical methodologies need 
to be developed to assess total model uncertainty and present results in a meaningful way. If uncertainties (however large) are explicitly reflected on model results, policy makers will still make decisions, but an inappropriate level of reliance on the results will be avoided (Pielke, 1998; Dabberdt and Miller, 2000).

The Monte-Carlo analysis is one of the commonly used methods for propagating input data errors through air quality models. It has been applied to models of different levels of sophistication, from Gaussian plume (Irwin et al., 1987) to complex photochemical codes (Hanna et al., 1998; 2001). This method enables an evaluation of the output of the model for many sets of combinations of the input parameters. These data sets are obtained by random sampling from the distribution assigned to each one of the uncertain input variables. Two important advantages of this statistical method are that it can be applied to a complete set of about 100 or more input parameters, and that it is widely used in the analysis of other environmental problems. On the other hand, it has the limitation that the estimates of uncertainty in the inputs are often based on informal processes (e.g. the professional judgement of the modeller), and that the cost of the method in terms of computer simulation time might be quite high, since a relatively large number $(>100)$ of model runs is preferable (Irwin et al., 1987; Hanna et al.. 1998).

Freeman et al. (1986) developed a theoretical formula for propagating input data errors through dispersion models. Results obtained using this formula were in good agreement with Monte-Carlo uncertainty estimates for unstable atmospheric conditions, but large inconsistencies were observed for neutral and stable conditions. These results also showed that even small uncertainties in the inputs might cause large uncertainties in the predictions.

Dabberdt and Miller (2000) used a probabilistic method for quantifying the uncertainty related to model predictions in an accidental release application. An ensemble set of 162 simulations was created by specifying a best estimate together with two additional values that bounded the likely range of uncertainty in estimating four input parameters (i.e. wind speed, wind direction, source strength, and plume rise). A best estimate together with a "second choice" was specified for atmospheric stability. Finally, contour concentration patterns (both deterministic and probabilistic) and histograms of the probability of occurrence of concentrations at specific receptor locations were produced to illustrate the uncertainty in the predictions.

Another way to estimate uncertainty in model predictions is by determining the input parameters to which the model in use is more sensitive. A so-called sensitivity analysis indicates how much of the overall uncertainty in the model predictions is associated with the individual uncertainty in each model input (McRae and Seinfeld, 1983). Sensitivity studies are not, strictly speaking, uncertainty analysis, since they do not combine the uncertainties of the model inputs to provide a realistic estimate of the uncertainty in the model output. Nevertheless, knowledge of the model's sensitivity to different variables is necessary in order to decide where emphasis should be placed in estimating total uncertainty (Hanna, 1988).

In this study, the sensitivity of three street canyon models (STREET, OSPM and AEOLIUS) to certain input variables and internal parameters was examined. Furthermore, practical methodologies for assessing model uncertainty were tested using air quality measurements from two street canyons in Paris.

\section{Experimental}

\section{1. Field measurements}

The data used in this study were collected during two field experiments carried out in Paris during winter (Bd. Voltaire, December 1998) and summer (Rue de Rennes, July 1999). The two sites were busy four lane streets with large pavements and uniform buildings lining up continuously on both sides. The aspect ratios (i.e. height/width) for Bd. Voltaire and Rue de Rennes were approximately equal to 0.8 and 1.1, respectively.

A number of traffic-related air pollutants were sampled during these campaigns. Hourly CO concentrations were measured at two roadside locations using an infrared analyser, and weekly benzene averages were obtained at different heights and distances from the kerb using diffusive samplers in both the canyons. The ensemble of these measurements revealed a significant spatial and temporal variability of air pollution in urban streets.

The background contribution to the roadside concentrations was derived from benzene measurements (in green spaces adjacent to the street monitoring sites) and an empirical relationship relating benzene to $\mathrm{CO}$ concentrations. Benzene background values ranged from $0.4 \mathrm{ppb}$ in Rue de Rennes to $1.3 \mathrm{ppb}$ in Bd. Voltaire (weekly averayes), while roadside values were from 2 to 6 times higher depending on the sampling location 
within the street (e.g. pavement, balcony, etc.). The experimental set-up and monitoring results from these two campaigns were presented and fully discussed elsewhere (Vardoulakis et al., 2002).

Synoptic meteorological information was obtained from three permanent weather stations operated by Meteo France. Two of them were located within Paris: in Montsouris park (anemometer height: $26 \mathrm{~m}$ ) and St. Jacques tower (anemometer height: $56 \mathrm{~m}$ ). The third station was located in Orly airport, at approximately $12 \mathrm{~km}$ distance from central Paris (anemometer height: $10 \mathrm{~m}$ ).

Hourly traffic data were obtained from automatic counters permanently operating in both the streets. The average traffic volumes in Bd. Voltaire and Rue de Rennes during measurements were 30,000 and 23,000 velh/day, respectively. The vehicle fleet composition was estimated from on site spot measurements.

\subsection{Traffic emissions}

The rate of release of emissions in the street was derived from the traffic volumes and the composite emission factor of the pollutant. Two different methods were applied for calculating $\mathrm{CO}$ emission factors: (a) The protocol used by Buckland and Middleton (1999), which was based on values specific to UK vehicles, and (b) the IMPACT road traffic emission model commercialised by ADEME (1998). This model uses COPERT II methodology (Ntziachristos and Samaras, 1997) to quantify fuel consumption and atmospheric releases of a specified vehicle fleet in a given year in France. The required input parameters are traffic composition, average vehicle speed, length and slope of the road segment of interest. In addition, the month of the year is used to estimate average ambient temperatures, which are further used for calculating evaporative and cold start/running emissions. The model provides default values for the average travelling distance and the fraction of this distance run with a cold engine in France.

The values estimated using both the methods were compared for consistency with $\mathrm{CO}$ emission factors specific to the French vehicle fleet reported in other recent studies (Touaty and Bonsang, 2000; Jones et al., 2000). All values were summarised in Table 1.

\section{Street canyon models}

Three parametric models (STREET, OSPM, and AEOLIUS) were used to simulate pollutant dispersion within the canyons. These relatively simple modeis (or variations of them) are likely to be used by local authorities or air quality networks in a variety of applications including air quality and traffic management, urban planning, population exposure studies, etc. In the following paragraphs, these models are briefly described and some of their empirical assumptions highlighted. References are provided for further in depth reading.

\subsection{STREET}

This empirical model calculates series of hourly concentrations at different receptor locations. The concentrations $(C)$ of the pollutant occurring on the roadside consist of two components, the urban background concentration $\left(C_{b}\right)$ and the concentration component $\left(C_{s}\right)$ due to vehicle emissions generated within the street:

$$
C=C_{s}+C_{b}
$$

The $C$, component was derived from a simple box model (Johnson et al., 1973). On the leeward side of the street, pollutant concentrations are given by the expression:

$$
C_{n}^{\prime \prime}=K \frac{Q}{\left(\sqrt{\left(x^{2}+z^{2}\right.}+h_{o}\right)\left(U+U_{s}\right)}
$$

where $K$ is an empirical constant parameter (usually given the value of 7), $Q$ is the rate of release of emissions in the streets, $x$ is the horizontal distance between the receptor and the centre of the nearest traffic lane, $z$ is the height of the receptor, $h_{t}$ is a constant that accounts for the height of initial pollutant dispersion (empirical value: $2 \mathrm{~m}), U$ is the roof-level wind speed, and $U_{S}$ is a constant that accounts for the additional air movement induced by vehicle traffic (empirical value: $0.5 \mathrm{~m} / \mathrm{s}$ ). 
On the windward side, the original expression given by Johnson et al. (1973) was revised by Dabberdt et al. (1973) to account for vertical decrease of concentrations due to entrainment of fresh air through the top of the canyon. The final equation was:

$$
C_{s}^{\prime \prime}=K \frac{Q}{W\left(U+U_{s}\right)} \frac{H-z}{H}
$$

where $H$ is the height and $W$ the width of the canyon. For parallel or near-parallel synoptic winds, the average of (2) and (3) should be calculated. Variations of this model have been widely used in scientific and engineering applications (Qin and Kot, 1993; Hoydysh and Dabberdt, 1988).

\subsection{OSPM and AEOLIUS}

AEOLIUS (the Full version) and OSPM are semi-empirical dispersion models based on the same mathematical formulation. They combine Gaussian plume theory with empirical box model techniques to calculate concentrations of exhaust gases in a street canyon assuming three different contributions: The contribution $\left(C_{d}\right)$ from the direct flow of pollutants from the source to the receptor, the recirculation component $\left(C_{r}\right)$ due to the flow of pollutants around the horizontal vortex generated within the recirculation zone of the canyon, and the urban background contribution $\left(C_{b}\right)$ :

$$
C=C_{d}+C_{r}+C_{b}
$$

A Gaussian plume model is used for the calculation of the direct contribution:

$$
C_{d}=\sqrt{\frac{2}{\pi}} \frac{Q}{W \sigma_{w}} F
$$

where $F$ is a factor depending on the synoptic wind, and $\sigma_{w}$ is the vertical velocity fluctuation due to mechanical turbulence generated by wind and vehicle traffic in the street. This is described by the relationship:

$$
\sigma_{n}=\sqrt{(\alpha u)^{2}+\sigma_{w n}^{2}}
$$

where $u$ is the street-level wind speed, $\alpha$ is a proportionality constant (given empirically the value of 0.1 ), and $\sigma_{w i}$ is the traffic-induced turbulence, defined as:

$\sigma_{u v l}=b\left(\frac{N \cdot V \cdot S^{2}}{W}\right)^{1 / 2}$

where $b$ is an aerodynamic drag coefficient (given empirically the value of 0.3 ), $N$ the number of vehicles using the street per time unit, $V$ the average vehicle speed, and $S^{2}$ the road surface occupied by a single vehicle, and $W$ the width of the canyon.

The contribution from the recirculation zone is calculated using a simple box model, which assumes that the pollutants are well mixed inside the box. This is expressed by the relationship:

$$
C_{r}=\frac{Q}{W} \frac{L_{r}}{\sigma_{w r} L_{1}+u_{1} L_{S 1}+u_{b} L_{S 2}}
$$

where $L_{r}, L_{l}, L_{S l}$ and $L_{S 2}$ are dimensions of the recirculation zone, which has the shape of a trapezium; $\sigma_{w t}$ is the ventilation velocity of the canyon expressed as: 
$\sigma_{w t}=\sqrt{(\lambda U)^{2}+F_{r o o f} \sigma_{w o}^{2}}$

where $U$ is the roof-level wind speed, and $\lambda$ and $F_{\text {rouf }}$ are proportionality constants given the value of 0.1 and 0.4 , respectively. The extension $L_{r}$ of the recirculation zone is defined as:

$L_{r}=F_{\text {rarler }} \cdot H \cdot r \sin \phi$

where $F_{\text {vartex }}$ is a proportionality constant given the value of $2, H$ is the height of the canyon, $r$ is a wind speed dependent factor reflecting the strength of the vortex, and $\phi$ the angle of the roof-level wind with respect to the street. The factor $r$ takes the values:

$$
\left\{\begin{array}{ccc}
r=1 & \text { if } & U>U_{\text {criical }} \\
\text { else } & & r=U / U_{\text {critical }}
\end{array}\right\}
$$

The critical velocity $U_{\text {criticut }}$ for the formation of the vortex in the street is empirically defined as $2 \mathrm{~m} / \mathrm{s}$. It should be noted that the width of the recirculation zone $L_{r}$ cannot exceed the width of the canyon in any case. The relation between street and roof-level winds in a regular canyon is given by:

$u=U \frac{\ln \left(h_{0} / z_{0}\right)}{\ln \left(H / z_{0}\right)}\left(1-F_{w i m d} \sin \phi\right)$

where $z_{\text {, }}$ is the surface roughness length of the area under consideration, $h_{0}$ the effective release height of car exhausts due to initial dispersion, and $F_{\text {sind }}$ an empirical constant given the value of 0.2 . The wind speed at rooflevel $(C)$ is calculated from the input wind $\left(U_{a}\right)$, which corresponds to a meteorological mast of generally different height, using the simple relationship:

$U=F_{m a x t} \cdot U_{a}$

The empirical parameter $F_{m u s}$ is given the value of 0.82 , which is derived from a logarithmic law similar to expression (12).

On the leeward side of the street, concentrations are calculated as the sum of the direct and recirculation contributions, while on the windward side only the direct contribution of emissions generated outside the recirculation zone are taken into account. If the recirculation zone extends throughout the whole canyon, then the windward concentrations are calculated from only the recirculation component. For near-parallel flow, emissions from outside the recirculation zone may contribute to the leeward concentrations. When the wind speed is near zero or parallel to the street axis, the concentrations on both sides of the canyon become equal. In all cases, the background contribution should be added to obtain the final result. A more detailed description of OSPM is given elsewhere (Berkowicz et al., 1997; Berkowicz, 2000).

OSPM has been applied to several street canyons in European and Asian cities (Berkowicz et al., 1996; Kukkonen et al., 200 I; Fu et al., 2000), while AEOLIUS has been mainly used in the UK (Buckland, 1998).

\section{Sensitivity analysis}

The above described models contain a number of constant parameters that have been empirically defined using experimental data. Although they might have a significant influence on model predictions and hence on the interpretation of the results, these constants have drawn relatively little attention so far. Comparing model results with experimental data, inappropriate values of such model constants might be falsely interpreted as unsatisfactory emission factors or meteorological input data (Buckland and Middleton, 1999).

STREET model includes three empirical parameters $K, h_{t}$ and $U_{S}$ (eq. 2 and 3 ), which have been adjusted to observed results. Johnson et al. (1973) derived initially the values of $K=7, h_{o}=2 \mathrm{~m}$ and $U_{s}=0.5 \mathrm{~m} / \mathrm{s}$, using data from the San Jose Street Canyon Experiment. The value of $K=7$ is presumably valid for canyons having 
$H / W \cong 1$, which is comparable to the aspect ratio of San Jose Street. A subsequent evaluation by Dabberdt et al. (1973) did not suggest dramatic variation in $K$ for two narrower canyons with $H / W$ of 1.5 and 2 in St. Luis. Yamartino and Wiegand (1986) kept the original values for $h_{0}$ and $U_{s}$, but allowed $K$ to rise to an optimal value of 10.2 in their evaluation of STREET model using measurements from Bonner Strasse $(H / W \cong 1)$, Cologne. In a research study in China, $K$ was given the value of 6 to simulate concentrations observed in an asymmetric street canyon in Guangzhou City (Qin and Kot, 1993). Finally, in a recent experiment in a street canyon in Buenos Aires $(H / W \cong 1$ ), best fit was obtained for $K=8$ (Bogo et al., 2001). Obviously, the value of $K$ weighs heavily on calculations, since it is directly proportional to the model output.

Buckland and Middleton (1999) and Manning et al. (2000) carried out sensitivity studies by varying a number of input variables and internal empirical parameters within AEOLIUS and assessing their impacts on the calculated $N_{\mathrm{x}}$ and $\mathrm{CO}$ concentrations, respectively. It was found that predicted values were almost linearly proportional to the emission factors and traffic flow. Canyon geometry did not significantly affect perpendicular concentrations for a given traffic flow and wind speed. However, that was not the case for parallel concentrations, which increased with canyon height due to the longer integration path before the plume escaped from the canyon, and decreased with canyon width. Increasing surface roughness was shown to enhance calculated concentration. Concentrations decreased at higher traffic speeds as the turbulent mixing increased, although this parameter did not appear to have a dramatic effect on the results. A change in the model coding of the extent of the vortex across the street produced only a minor change in leeward concentrations. Buckland and Middleton (1999) altered the model to let the user specify whether the wind speed was measured at $10 \mathrm{~m}$ height (as it is usually the case in airports) or at roof-level. That had only a small effect on the results. A constant value $(0.1 \mathrm{~m} / \mathrm{s})$ was tested for replacing equation (7), which calculates vehicle-induced turbulence. This generated much larger concentrations. Finally, it was shown that the assumed magnitudes of arbitrary model constants such as $b$ or $\lambda$ had a marked effect on calculated concentrations.

\section{t. I. Internal parameters}

In the present study, a sensitivity analysis was performed to determine the importance of various internal model parameters on the output of OSPM. Minor modifications were made in the code of the model, so as to enable the

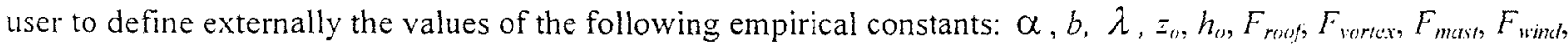
and $U_{\text {criticul }}$. The values of these parameters were then perturbed to observe the effects on the predicted $\mathrm{CO}$ concentrations for perpendicular (i.e. leeward and windward) and parallel wind conditions. For the sensitivity runs. the characteristics of the regular canyon of Rue de Rennes were used, while the input wind speed was maintained constant $(3.5 \mathrm{~m} / \mathrm{s})$. A large number of diagrams similar to Fig. 1, 2, and 3 were produced (not shown).

As expected, most of the conclusions from the previous sensitivity analysis for AEOLIUS were also valid for OSPM. An increase in surface roughness $\left(z_{t \prime}\right)$ enhanced parallel and leeward concentrations, but had no effect on windward concentrations (Fig. 1). Larger $z_{t}$ values would be expected to reduce the street-level wind, but increase the turbulence within the canyon (Manning et al., 2000). However, OSPM simply reduced the streetlevel wind, which in turn lowered the mechanical turbulence (eq. 6) and increased concentrations.

The effective release height $\left(h_{0}\right)$ had the opposite effect on the calculated results. This parameter, which is user defined in AEOLIUS but originally coded inside OSPM $\left(h_{0}=2\right)$, also represents the height of the simulated roadside receptor. In this analysis, parallel and leeward concentrations decreased with $h_{t}$, while the values on the windward side remained constant.

OSPM perpendicular concentrations were proved to be quite sensitive to the value of $\lambda$ (Fig. 2), which sets the rate at which material is dispersed out of the top of the canyon, but there was no effect on the parallel concentrations. It should be noted that $\lambda$ might be sensitive to seasonal weather variations, since atmospheric stability can play a role in the ventilation of street canyons. Parameter $\alpha$ had no marked effect on OSPM results.

As it can be observed in Fig. 3, the aerodynamic parameter $b$ had certain influence on the parallel and leeward concentrations, due to the role of cruising vehicles in the calculation of the mechanical turbulence in the street. For the range of values tested, $b$ had no significant effect on the windward concentrations. When assigning empirical values to $b$, it should be remembered that the aerodynamic drag of cars is likely to diminish in the future due to improvements in the design of new vehicles. 
The influence of the coefficient $F_{\text {mast }}$ (eq. 13) was found to be of minor importance. Nevertheless, OSPM code was here slightly modified by replacing relationship (13) with the following:

$$
U=U_{"} \frac{\ln \left(\frac{H}{z_{o}}\right)}{\ln \left(\frac{H_{u}}{z_{0}}\right)}
$$

which allows the user to specify the height of the anemometer $\left(H_{\alpha}\right)$; this information may be easily and accurately obtained from weather station operators.

Altering the value of $F_{\text {vertux }}$ (eq. 10 ), a significant effect on leeward concentrations was produced due to changes in the dimensions of the vortex. For example, a change from the standard value of 2 to the value of 1 resulted in a $31 \%$ decrease in leeward CO concentrations. No marked effect was observed on the windward and parallel concentrations for such a change.

The $F_{\text {utut }}$ coefficient (eq. 12) had a small influence only on leeward concentrations, since it only affects the dispersion of pollutants coming directly from the source. An increase from the standard value of 0.2 to the value of 0.4 produced $11 \%$ higher leeward concentrations. Finally, the coefficient $F_{\text {ruof }}$ (eq. 9) and the critical wind speed $U_{\text {critictit }}$ for vortex formation (eq. 11) had no marked effect on the model results for the range of tested values.

\subsection{Emission factors}

The CO emission factor calculated using IMPACT was $25 \%$ higher than the value calculated according to Buckland and Middleton (1999) for Rue de Rennes during the summer campaign. For Bd. Voltaire, the IMPACT model estimate was $180 \%$ higher than the value calculated using Buckland and Middleton's (1999) methodology (Table 1). This large discrepancy between the two methods may be justified by the fact that IMPACT also added the very significant cold start/running emissions (during winter) to the hot running values. Cold start/running emissions are indeed expected to be important for urban driving conditions, because of the relative large number of short trips carried out with cold engines, especially in winter. In an on-road experiment in Belgium (De Vlieger, 1997), it was found that the average CO vehicle emissions measured during the cold phase were 4 to 40 times higher than emissions with a hot start. Other experiments showed significant seasonal differences in cold starts due to ambient temperature variations (Mensink et al., 2000).

Furthermore, it has been reported that emissions obtained from aggressive driving can be up to four times higher than those obtained from normal driving (De Vlieger, 1997), and that emission factors typically increase by a factor up to ten during congestion compared to smooth driving conditions (Sjodin et al., 1998). Finally, it should be remembered that the vast majority of fleet emissions come from a small number of poorly maintained vehicles (Singh and Huber, 2000).

The selection of the appropriate emission model or methodology is crucial, since model predictions are almost linearly proportional to the estimated emission factors. The methodology implemented in IMPACT may be seen as more suitable for the present study (especially for the winter campaign) than the one used by Buckland and Middleton (1999), because it takes into account the average vehicle speed and the seasonal influences in vehicle start/running emissions.

\subsection{Meteorological data}

Meteorological data obtained simultaneously at different weather stations located within few kilometre distances from each other might differ significantly, especially for short averaging periods. When the first street canyon models were developed few decades ago, it was assumed that the local roof-level wind information needed as an input would not be generally available, and airport wind data would have to be used. For this reason, empirical expressions relating airport and local roof-level winds were derived (Johnson et al., 1973).

Exploring the sensitivity of AEOLIUS to wind data from different sources, Manning et al. (2000) observed that model concentrations were significantly lower when airport rather than local winds were used. That was in 
agreement with the present study, which showed that simulations carried out using wind data from Orly airport generally produced lower and less accurate predictions compared to those produced using urban wind data.

Nowadays, there is at least one weather station permanently operating within every European capital. It is, therefore, suggested that wind information from airports be avoided, when suitable urban meteorological measurements (obtained under the same quality criteria) are available for running street canyon models.

\section{Uncertainty analysis}

\subsection{Statistical evaluation}

STREET, OSPM and AEOLIUS were initially used in a traditional manner to create time series of CO best estimates in Bd. Voltaire and Rue de Rennes. Instead of defining a priori uncertainty ranges in different input variables, three independent meteorological data sets and three different emission factors (as described in section 2) were used to create ensemble sets of 27 model realisations for each time step.

In the same manner, ensemble sets of 27 model realisations were also created for benzene. In that case, instead of time series, weekly averages corresponding to different receptor locations in the streets were calculated following a practical methodology described by Vardoulakis et al. (2002).

Four statistical evaluation measures were then applied to quantify the differences between predicted and observed concentrations (Yadav and Sharan, 1996): (1) The fractional bias (FB), which provides information on the tendency of the model to over-estimate or under-estimate the observed concentrations (negative values show over-predictions), (2) the normalised mean square error (NMSE), which provides information on the overall deviations between predicted and observed concentrations, (3) the correlation coefficient, which describes the degree of association between variables, and (4) the fraction of predictions within a factor of two (FAC2).

The results of this analysis for 1- and 8-hour CO predictions in Rue de Rennes are presented in Table 2. It can be seen that for longer averaging times (i.e. 8 hours), the performance of all three models was enhanced. That was expected, since the uncertainty attributed to the turbulent atmospheric processes generally decreases as averaging times increase.

Although there were no dramatic differences in the statistics for the different trials, it might be concluded that OSPM performed slightly better than the other two models in the case of Rue de Rennes. For OSPM, the correlation coefficient ranged from 0.68 to 0.77 for 1 -hour averages and from 0.81 to 0.89 for 8 -hour averages. For the same model, the FAC2 values ranged from 0.66 to 0.78 for I-hour averages and from 0.80 to 0.92 for 8 hour averages. The optimum FB values (i.e. near zero) were observed for simulations carried out with emission factors calculated according to Buckland and Middleton (1999) and wind data obtained from Montsouris station.

When the same statistical measures were applied to the Bd. Voltaire data, STREET seemed to give slightly better predictions than the other two models. For STREET, the correlation coefficients ranged from 0.74 to 0.75 for 1hour averages and from 0.96 to 0.97 for day averages (night measurements were not available in Bd. Voltaire). In that case, it was revealed that emission factors calculated using IMPACT gave the best agreement (FB $\cong 0$ for

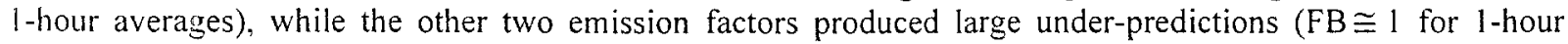
averages). A reason for this was probably the fact that IMPACT accounted also for the very significant cold start/running emissions in Bd. Voltaire, an urban environment with many parking spaces, during winter. The use of urban meteorological data was again found to improve the model results.

Finally, some significant discrepancies between OSPM and AEOLIUS predictions were identified (e.g. NMSE and FAC2 values in Table 2), despite the fact that both the models are based on the same formulation. This is probably due to certain differences in coding, parameterisation and data pre-processing techniques between the two models.

\subsection{Concentration ranges using three models}

A comparison of statistical performance measures helps to determine if one model or input data set is better than another for a specific application. In certain cases, however, model results may deviate quite significantly (especially for short averaging times), without this being reflected on the overall statistics. 
Medians together with maximum and minimum concentrations were calculated for each ensemble set of 27 model realisations corresponding to a specific time and location within the two streets in Paris. The extreme concentrations were thought to give a rough estimate of model uncertainty in the predictions (Vardoulakis at al., 2001 ). As it can be seen in Fig. 4 and 5, approximately $92 \%$ and $95 \%$ of CO observations in Bd. Voltaire and Rue de Rennes, respectively, lie within the predicted concentration ranges. In the same manner, error bounds were also attached to 8-hour mean $\mathrm{CO}$ concentrations (Fig. 6), since CO standards are written as 8-hour averages.

The same method was used to obtain a rough estimate of model uncertainty in benzene predictions corresponding to different sampling locations within the two canyons. As it can be seen in Fig. 7 and 8, all observed benzene concentrations (weekly averages) lie within the estimated error bounds.

An alternative method using fuzzy numbers to treat predictions from more than one model has been recently proposed (Fisher and Ireland, 2001). This method, which provides probability weightings on model predictions, may be applied at a later stage of an air quality assessment, if a more advanced interpretation of the modelling results is needed.

As far as the choice of models is concerned, the intention was to define the appropriate degree of sophistication for the specific applications, so as to minimise uncertainties. It might be incorrectly assumed that the total uncertainty in predictions always decreases as the complexity of a model increases. This is only true for the uncertainty attributed to errors in the physical description of the model domain (e.g. incorrect assumptions, oversimplifications, etc.). On the other hand, sophisticated models require a larger amount of input information, which inevitably introduces a larger data uncertainty component in their calculations.

\subsection{Concentration ranges using one model}

Assuming that there is only one model and one input data set available, it is still possible to have a rough estimate of uncertainty in model predictions. This can be achieved by assigning a best estimate together with two additional values that may bound the likely range of uncertainty related to certain internal model parameters. In

this case. $\lambda, b$, and $z_{1}$, were selected because they were found to have a significant effect on OSPM results (see section 4.1).

Using the meteorological data from Montsouris station and the emission factors calculated according to Buckland and Middleton (1999), an ensemble set of 27 OSPM simulations was again created by only varying the values of $b$ and $z_{n}$ by $33 \%$ and the value of $\lambda$ by $50 \%$, as shown in Table 3 . The "max" and "min" values assigned to the three selected parameters were in a reasonable agreement with values previously tested by Buckland and Middleton (1999), and Manning et al. (2000).

Although the number of simulations was here the same as in the examples of section 5.2, the estimated concentration ranges were significantly narrower (Fig. 9). As a result, more than $20 \%$ of the observed CO concentrations fell outside the estimated concentration ranges.

A more rigorous approach would require that probability functions be developed for each "sensitive" input or internal model parameter, and that these be randomly sampled to obtain improved ensemble sets (Dabberdt and Miller, 2000). On the other hand, this approach would require a much larger number of model runs, which would increase the time and consequently the cost of simulations. Furthermore, it is more important to specify the width rather than the shape of any probability functions describing the uncertainty of the variables (Alcamo and Bartnicki, 1987).

Finally, it should be stressed that this single model approach can be applied only if the user has access to a number of empirical model parameters, which is usually not the case since they are often coded inside the model.

\section{5.t. C'omparison with regulatory standards}

It has already been discussed that the traditional method of applying air quality models disregards uncertainty. There is therefore a risk of ending up with a misleading classification of urban environments in only two, "yes" or "no" polluted categories. What is really needed is a probabilistic comparison of predicted values against regulatory limits that takes into account best model estimates as well as their related error bounds. 
Having calculated the concentration ranges (section 5.2), a probabilistic method (Ramsey and Argyraki, 1997) was adopted for assessing the compliance of different kerbside locations with an ambient air quality standard. According to this method, the predicted benzene concentrations (Fig. 7 and 8) were classified in four different categories with respect to the proposed EU limit value of $5 \mu \mathrm{g} / \mathrm{m}^{3}$ (i.e. $1.66 \mathrm{ppb}$ ): (a) "exceeding the limit" if the predicted minimum value for one location was above the threshold, (b) "probably exceeding" if the predicted median was above the limit while the minimum was below, (c) "possibly exceeding" if the predicted median was below the limit but the maximum above, and finally (d) "not exceeding" if the predicted maximum lay below the threshold. According to this classification, locations 1, 3, and 4 in Fig. 7 were found to exceed the EU threshold, while location 2 was probably exceeding the same limit value. In Fig. 8, sampling locations $1,2,3,4,5$, and 10 were exceeding the threshold, location 11 was probably exceeding, locations $6,7,8$, and 12 were possibly exceeding, and finally only location 9 was found not to exceed the limit.

Although the averaging times in Fig. 7 and 8 do not directly correspond with the EU standard value for benzene (i.e. annual average), these examples show a precautionary way of applying a discrete air quality criterion. It should be remembered that it is possible to relate short-term averages to annual standards (e.g. for benzene) by means of an appropriate surrogate (e.g. $\mathrm{CO}$ ) measured throughout the year. An alternative approach would allow for a certain degree of tolerance to be associated with the criterion itself, instead of attaching error bounds to the predictions.

\section{Conclusions}

The sensitivity of regulatory street canyon models to certain input variables (i.e. emission factors and meteorological data) and internal parameters was studied. Emphasis was put on the sensitivity of OSPM to the full set of empirical constants coded inside the model. Furthermore, practical methodologies for obtaining first estimates of model uncertainty were tested using air quality data from two field experiments in Paris.

Two different approaches were used to create ensemble sets of 27 realisations, which were then used to derive best pollutant (i.e. $\mathrm{CO}$ and benzene) concentration estimates and related error bounds. Statistical techniques were applied to evaluate the performance of each simulation. It was shown that the use of wind information obtained from urban monitoring stations optimised the application of models. Large uncertainties in vehicle emission factors were identified.

A probabilistic method for assessing air pollution in urban streets was proposed. Although sophisticated statistical and error propagation techniques were avoided, the above methodologies certainly increased to some extent the complexity of the simulations. It is, however, believed that they can contribute to reducing the risk of misinterpreting modelling results and making erroneous management decisions.

It should be finally noted that uncertainties related to air quality measurements (e.g. sampling and analytical errors) were not taken into consideration. These uncertainties, though generally smaller than model uncertainties, may have some implications in decision making, if the predicted concentrations are close to an air quality standard.

\section{Acknowledgements}

This work was inspired by lectures and presentations delivered by Drs. M. H. Ramsey and R. Colvile (University of London), who are hereby gratefully acknowledged. The authors would like to extend their appreciation to Jean-Claude Pinard and Armelle Frezier (INERIS) for their technical assistance, and Drs. R. Berkowicz (NERI) and D. Middleton (UK Met. Office) for discussing the models. Our appreciation is also given to the anonymous referee who helped to improve the clarity of the article. 


\section{References}

ADEME, 1998. Emissions de polluants et consommations liées à la circulacion routière. Paris, ADEME.

Alcamo J., Bartnicki J., 1987. A framework for error analysis of a long-range transport model with emphasis on parameter uncertainty. Atmospheric Environment 21,2121-2131.

Berkowicz R., 2000. OSPM - A parameterised street pollution model. Environmental Monitoring and Assessment 65, 323-331.

Berkowicz R., Hertel O., Larsen S.E., Sorensen N.N., Nielsen M., 1997. Modelling traffic pollution in streets. NERI, Roskilde, Denmark.

Berkowicz R., Palmgren F., Hertel O., Vignati E., 1996. Using measurements of air pollution in streets for evaluation of urban air quality - meteorological analysis and model calculations. The Science of the Total Environment 189/190, 259-265.

Bogo H., Gomez D.R., Reich S.L., Negri R.M., San Roman E., 2001. Traffic pollution in a downtown site of Buenos Aires City. Atmospheric Environment 35, 1717-1727.

Buckland A.T., 1998. Validation of a street canyon model in two cities. Environmental Monitoring and Assessment 52, 255-267.

Buckland A.T., Middleton D.R., 1999. Nomograms for calculating pollution within street canyons. Atmospheric Environment 33, 1017-1036.

Dabberdt W.F., Ludwig F.L., Johnson W.B., 1973. Validation and applications of an urban diffusion model for vehicular pollutants. Atmospheric Environment 7, 603-618.

Dabberdt W.F.. Miller E., 2000. Uncertainty, ensembles and air quality dispersion modeling: applications and challenges. Atmospheric Environment 34, 4667-4673.

De Vlieger 1., 1997. On-board emission and fuel consumption measurement campaign on petrol-driven passenger cars. Atmospheric Environment 31, 3753-3761.

Fisher B.E.A, Ireland M., 2001. Using fuzzy numbers to treat predictions from more than one model. 25th NATO/CCMS International Technical Meeting on Air Pollution and its Application, Louvain-la-Neuve, Belgium.

Freeman D.L., Egami R.T., Robinson N.F., Watson J.G., 1986. A method for propagating measurement uncertainties through dispersion models. Journal of Air Pollution Control Association 36, 246-253.

Fu L.. Hao J., Hertel O., Berkowicz R., 2000. Modeling traffic-related air pollution in street canyons of Beijing. J. Air \& Waste Manage. Assoc. 50, 2060-2066.

Hanna S.R., 1988. Air Quality Model Evaluation and Uncertainty. Journal of Air Pollution Control Association 38, 406-412.

Hanna S.R.. Chang J.C., Fernau M.E., 1998. Monte Carlo estimates of uncertainties in predictions by a photochemical grid model (UAM-IV) due to uncertainties in input variables. Atmospheric Environment 32. 3619-3628.

Hanna S.R., Lu Z., Frey H.C., Wheeler N., Vukovich J., Arunachalam S., Fernau M., Hansen D.A., 2001. Uncertainties in predicted ozone concentrations due to input uncertainties for the UAM-V photochemical grid model applied to the July 1995 OTAG domain. Atmospheric Environment 35, 891903.

Hoydysh W.G., Dabberdt W.F., 1988. Kinematics and dispersion characteristics of flows in asymmetric street canyons. Atmospheric Environment 22, 2677-2689.

Irwin J.S., Rao S.T., Petersen W.B., Turner D.B., 1987. Relating error bounds for maximum concentration estimates to diffusion meteorology uncertainty. Atmospheric Environment 21, 1927-1937.

Johnson W.B., Ludwig F.L., Dabberdt W.F., Allen R.J., 1973. An urban diffusion simulation model for carbon monoxide. Journal of the Air Pollution Control Association 23, 490-498.

Jones S.G., Fisher B.E.A., Gonzalez-Flesca N., Sokhi R., 2000. The use of measurement programmes and models to assess concentrations next to major roads in urban areas. Environmental Monitoring and Assessment 64, 531-547.

Kukkonen J., Valkonen E., Walden J., Koskentalo T., Aarnio P., Karppinen A., Berkowicz R., Kartastenpää R., 2001. A measurement campaign in a street canyon in Helsinki and comparison of results with predictions of the OSPM model. Atmospheric Environment 35, 231-243.

Manning A.J., Nicholson K.J., Middleton D.R., Rafferty S.C., 2000. Field study of wind and traffic to test a street canyon pollution model. Environmental Monitoring and Assessment 60, 283-313.

McRae G.J., Seinfeld J.H., 1983. Development of a second-generation mathematical model for urban air pollution - II. Evaluation of model performance. Atmospheric Environment 17, 501-522.

Mensink C., De Vlieger I., Nys J., 2000. An urban transport emission model for the Antwerp area. Atmospheric Environment 34, 4595-4602.

Ntziachristos L., Samaras Z., 1997. COPERT II: Computer programme to calculate emissions from road transport - User's manual. European Environment Agency, European Topic Center on Air Emissions. 
Pielke R.A., 1998. The need to assess uncertainty in air quality evaluation. Atmospheric Environment 32, 1467. 1468.

Qin Y., Kot S.C., 1993. Dispersion of vehicular emission in street canyons, Guangzhou city, South China (P.R.C.). Atmospheric Environment 27B, 283-291.

Ramsey M.H., Argyraki A., 1997. Estimation of measurement uncertainty from field sampling: implications for the classification of contaminated land. The Science of the Total Environment 198, 243-257.

Singh R.B., Huber A.H., 2000. Development of a microscale emission factor model for CO for predicting realtime motor vehicle emissions. J. Air Waste Manage. Assoc. 50, 1980-1991.

Sjodin A., Person K., Andreasson K., Arlander B., Galle B., 1998. On-road emission factors derived from measurements in a traffic tunnel. Int. J. Vehicle Design 20, 147-158.

Touaty M., Bonsang B., 2000. Hydrocarbon emissions in a highway tunnel in the Paris area. Atmospheric Environment, 985-996.

Vardoulakis S., Fisher B.E.A, Gonzalez-Flesca N., Pericleous K., 2001. Estimates of uncertainty in urban air quality model predictions. 25th NATO/CCMS International Technical Meeting on Air Pollution and its Application, Louvain-la-Neuve, Belgium.

Vardoulakis S., Gonzalez-Flesca N., Fisher B.E.A., 2002. Assessment of traffic-related air pollution in two street canyons in Paris: implications for exposure studies. Atmospheric Environment 36 (6), 1025-1039.

Yadav A.K., Sharan M., 1996. Statistical evaluation of sigma schemes for estimating dispersion in low wind conditions. Atmospheric Environment 30 (14), 2595-2606.

Yamartino R.J., Wiegand G., 1986. Development and evaluation of simple models for the flow, turbulence and pollutant concentration fields within an urban street canyon. Atmospheric Environment 20, 2137-2156. 


\section{CAPTIONS}

Table 1: CO emission factors from road transport in the region of Paris.

Table 2: Statistical evaluation of 27 model simulations for predicting 1- and 8-hour CO concentrations in Rue de Rennes, using three models (OSPM, STREET, and AEOLIUS), three wind data sets (from M: Montouris park, S: St-Jacques tower, and O: Orly airport), and three emission factors (according to 1: Buckland and Middleton, 2: IMPACT-ADEME, 3: Touaty and Bonsang).

Table 3: Values of three internal OSPM parameters ( $b$ : aerodynamic drag coefficient, $z_{0}$ : surface roughness length, and $\lambda$ : canyon ventilation coefficient) used for creating an ensemble set of 27 simulations.

Fig. 1: OSPM sensitivity to the surface roughness length $\left(z_{0}\right)$ for three different wind regimes

Fig. 2: OSPM sensitivity to the canyon ventilation coefficient $(\lambda)$ for three different wind regimes

Fig. 3: OSPM sensitivity to the aerodynamic drag coefficient $(b)$ for three different wind regimes

Fig. 4: Time series of best 1 -hour CO estimates (median), error bounds (max, $\min$ ), and observed concentrations in Bd. Voltaire produced using three models.

Fig. 5: Time series of best 1-hour CO estimates (median), error bounds (max, min), and observed concentrations in Rue de Rennes produced using three models.

Fig. 6: Time series of best 8-hour CO estimates (median), error bounds (max, $\min$ ), and observed concentrations in Rue de Rennes produced using three models.

Fig. 7: Best weekly benzene estimates (median), error bounds (max, min), and observed concentrations at different receptor locations in $\mathrm{Bd}$. Voltaire, produced using three models. The dashed line shows the EU proposed limit value for benzene (1.66 ppb).

Fig. 8: Best weekly benzene estimates (median), error bounds (max, min), and observed concentrations at different receptor locations in Rue de Rennes produced using three models. The dashed line shows the EU proposed limit value for benzene (1.66 ppb).

Fig. 9: Time series of best 1-hour CO estimates (median), error bounds (max, min), and observed concentrations in Rue de Rennes produced using one model (OSPM). 
Table 1

\begin{tabular}{lcccc}
\hline \multicolumn{1}{c}{ Author } & \multicolumn{3}{c}{ CO emission factors $(\mathrm{g} / \mathrm{km}$ veh) } \\
\cline { 3 - 4 } Buckland and Middleton (1999) & Bd. Voltaire & Rue de Rennes & Paris \\
IMPACT (ADEME, 1998) & 20.29 & 8.73 & \\
Touaty and Bonsang (2000) & & 10.91 & \\
Jones et al. (2000) & & & 8.11 \\
\hline
\end{tabular}


Table 2

\begin{tabular}{|c|c|c|c|c|c|c|c|c|}
\hline $\begin{array}{l}\text { Statistical evaluation } \\
\text { Ideal value }\end{array}$ & Correlatic & oefficient & Fracti & Bias & & & & \\
\hline Averaging time & 1 hour & 8 hour & 1 hour & 8 hour & 1 hour & 8 hour & 1 hour & 8 hour \\
\hline OSPM (M1) & 0.68 & 0.82 & 0.10 & 0.00 & 0.38 & 0.15 & 0.76 & 0.88 \\
\hline OSPM (M2) & 0.68 & 0.82 & -0.12 & -0.15 & 0.42 & 0.21 & 0.78 & 0.84 \\
\hline OSPM (M3) & 0.68 & 0.82 & 0.17 & 0.14 & 0.40 & 0.18 & 0.73 & 0.88 \\
\hline OSPM (S1) & 0.77 & 0.89 & 0.13 & 0.11 & 0.31 & 0.12 & 0.78 & 0.88 \\
\hline OSPM (S2) & 0.77 & 0.89 & -0.09 & -0.11 & 0.34 & 0.15 & 0.76 & 0.88 \\
\hline OSPM (S3) & 0.77 & 0.89 & 0.21 & 0.19 & 0.33 & 0.14 & 0.76 & 0.92 \\
\hline OSPM (01) & 0.71 & 0.81 & 0.19 & 0.16 & 0.41 & 0.23 & 0.71 & 0.80 \\
\hline OSPM (O2) & 0.71 & 0.81 & -0.03 & -0.06 & 0.41 & 0.25 & 0.78 & 0.84 \\
\hline OSPM (O3) & 0.71 & 0.81 & 0.27 & 0.24 & 0.45 & 0.26 & 0.66 & 0.80 \\
\hline STREET (M1) & 0.72 & 0.83 & 0.40 & 0.38 & 0.55 & 0.34 & 0.65 & 0.72 \\
\hline STREET (M2) & 0.72 & 0.83 & 0.18 & 0.17 & 0.42 & 0.20 & 0.78 & 0.84 \\
\hline STREET (M3) & 0.72 & 0.83 & 0.47 & 0.45 & 0.64 & 0.37 & 0.57 & 0.64 \\
\hline STREET (S1) & 0.76 & 0.89 & 0.54 & 0.52 & 0.72 & 0.45 & 0.49 & 0.44 \\
\hline STREET (S2) & 0.76 & 0.89 & 0.33 & 0.31 & 0.46 & 0.21 & 0.65 & 0.76 \\
\hline STREET (S3) & 0.76 & 0.89 & 0.61 & 0.59 & 0.84 & 0.56 & 0.43 & 0.40 \\
\hline STREET (O1) & 0.64 & 0.74 & 0.58 & 0.56 & 0.95 & 0.67 & 0.44 & 0.48 \\
\hline STREET $(\mathrm{O} 2)$ & 0.64 & 0.74 & 0.37 & 0.35 & 0.67 & 0.41 & 0.57 & 0.60 \\
\hline STREET (O3) & 0.64 & 0.74 & 0.65 & 0.63 & 1.08 & 0.79 & 0.38 & 0.44 \\
\hline AEOLIUS (M1) & 0.64 & 0.80 & 0.11 & 0.07 & 0.45 & 0.19 & 0.59 & 0.68 \\
\hline AEOLIUS (M2) & 0.66 & 0.81 & -0.11 & -0.14 & 0.47 & 0.23 & 0.56 & 0.64 \\
\hline AEOLIUS (M3) & 0.66 & 0.80 & 0.19 & 0.15 & 0.46 & 0.20 & 0.59 & 0.72 \\
\hline AEOLIUS (S1) & 0.71 & 0.86 & 0.16 & 0.13 & 0.43 & 0.17 & 0.49 & 0.64 \\
\hline AEOLIUS (S2) & 0.71 & 0.87 & -0.06 & -0.09 & 0.47 & 0.20 & 0.46 & 0.60 \\
\hline AEOLIUS (S3) & 0.71 & 0.87 & 0.24 & 0.21 & 0.45 & 0.18 & 0.49 & 0.64 \\
\hline AEOLIUS (O1) & 0.67 & 0.80 & 0.40 & 0.36 & 0.64 & 0.36 & 0.51 & 0.56 \\
\hline AEOLIUS (O2) & 0.67 & 0.80 & 0.19 & 0.15 & 0.48 & 0.24 & 0.57 & 0.68 \\
\hline AEOLIUS (O3) & 0.67 & 0.80 & 0.47 & 0.44 & 0.72 & 0.43 & 0.47 & 0.56 \\
\hline
\end{tabular}


Table 3

\begin{tabular}{lllccc}
\hline \multicolumn{2}{c}{ Coefficients } & & \multicolumn{3}{c}{ Estimated values } \\
\cline { 5 - 6 } & & & $\max$ & standard & $\min$ \\
\cline { 5 - 6 } Aerodynamic drag & $(b)$ & 0.40 & & 0.30 & 0.20 \\
Surface roughness & $\left(z_{0}\right)$ & 0.80 & 0.60 & 0.40 \\
Canyon ventilation & $(\lambda)$ & 0.15 & 0.10 & 0.05 \\
\hline
\end{tabular}




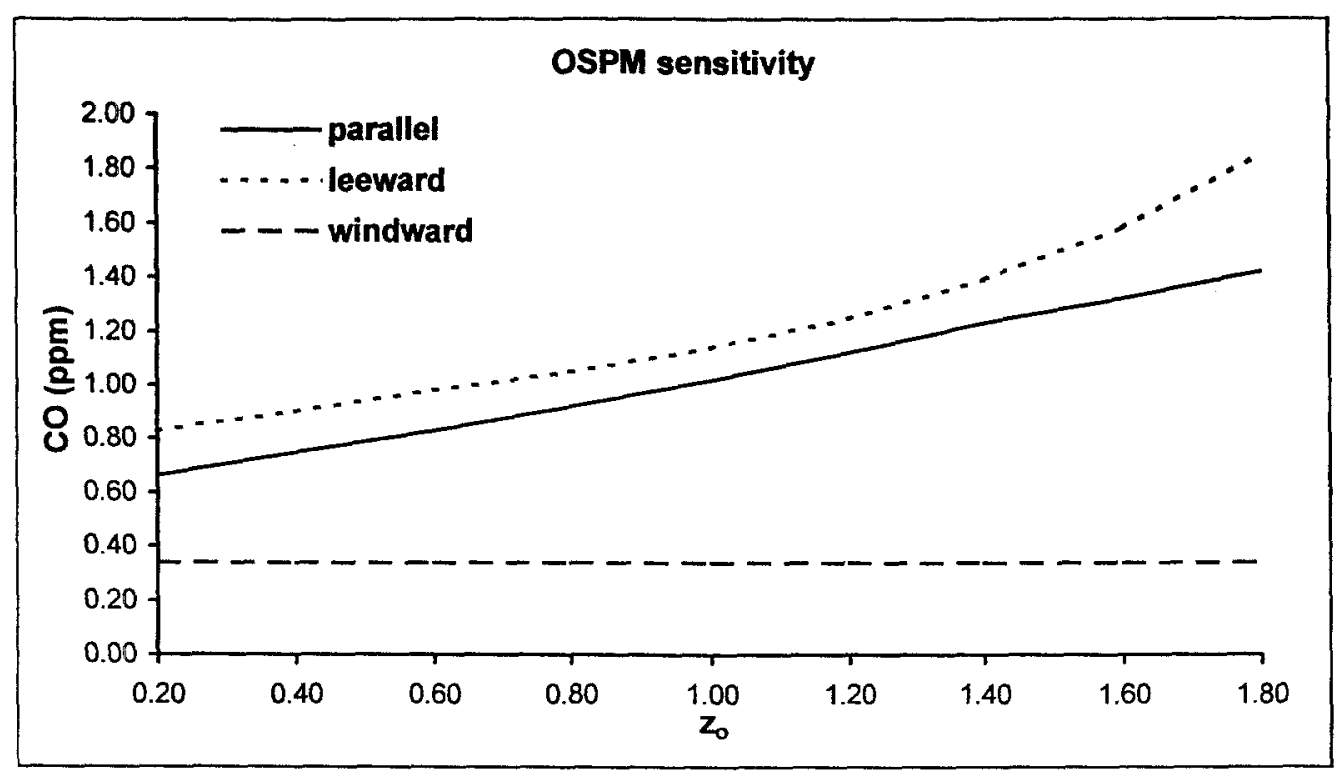

Fig. 1

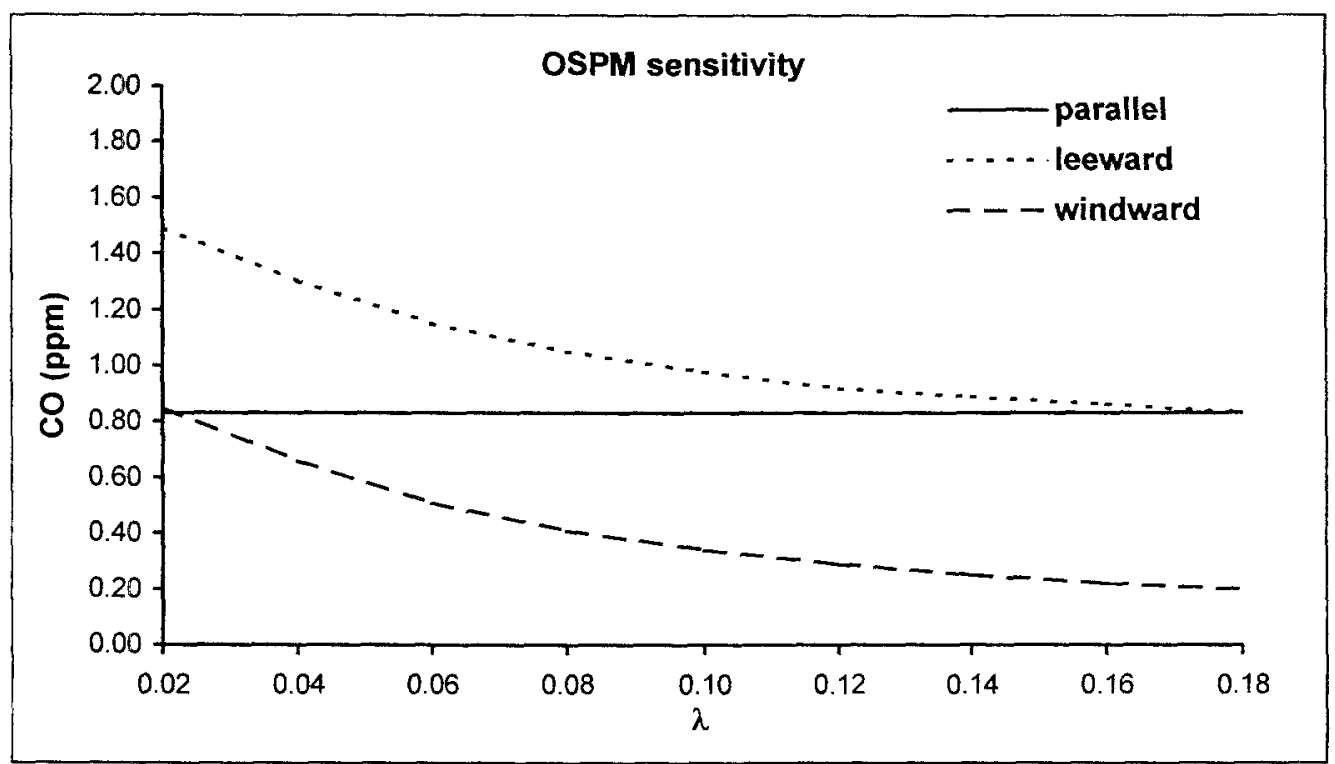

Fig. 2

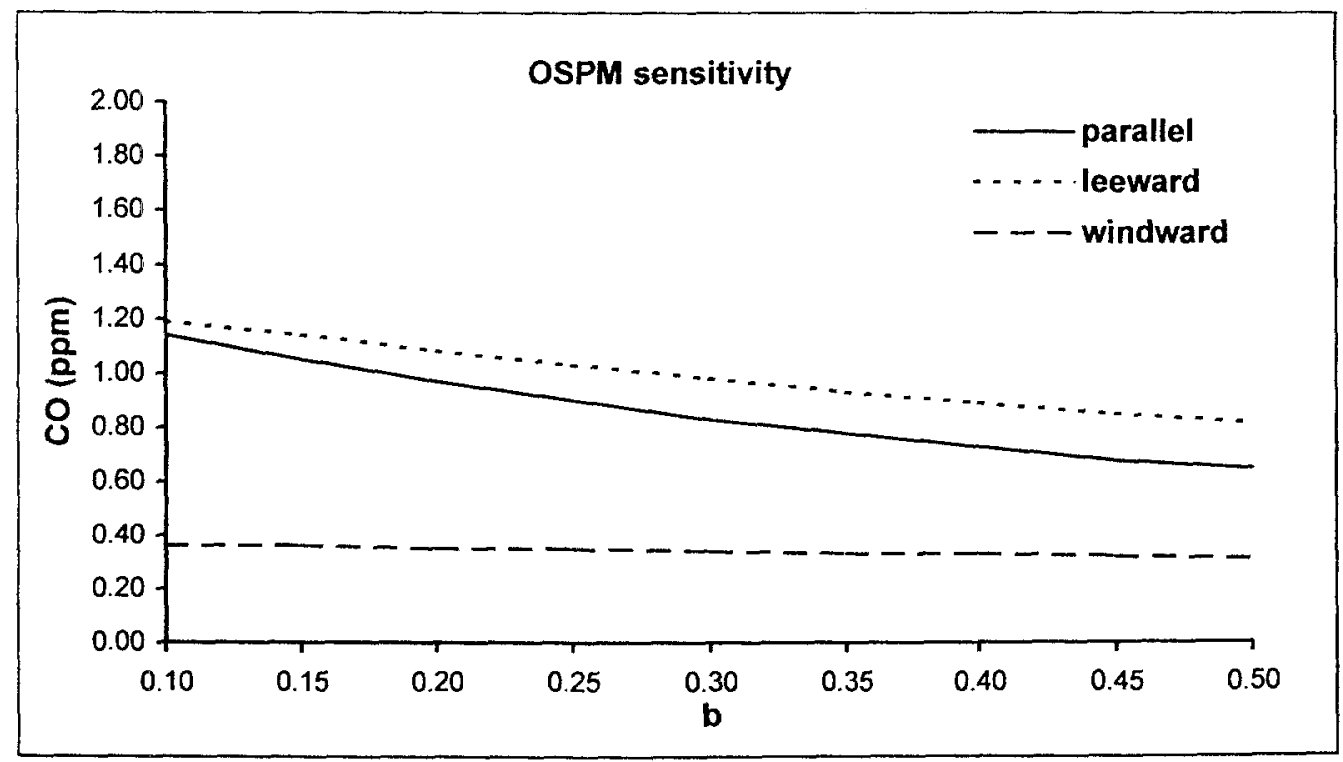

Fig. 3 


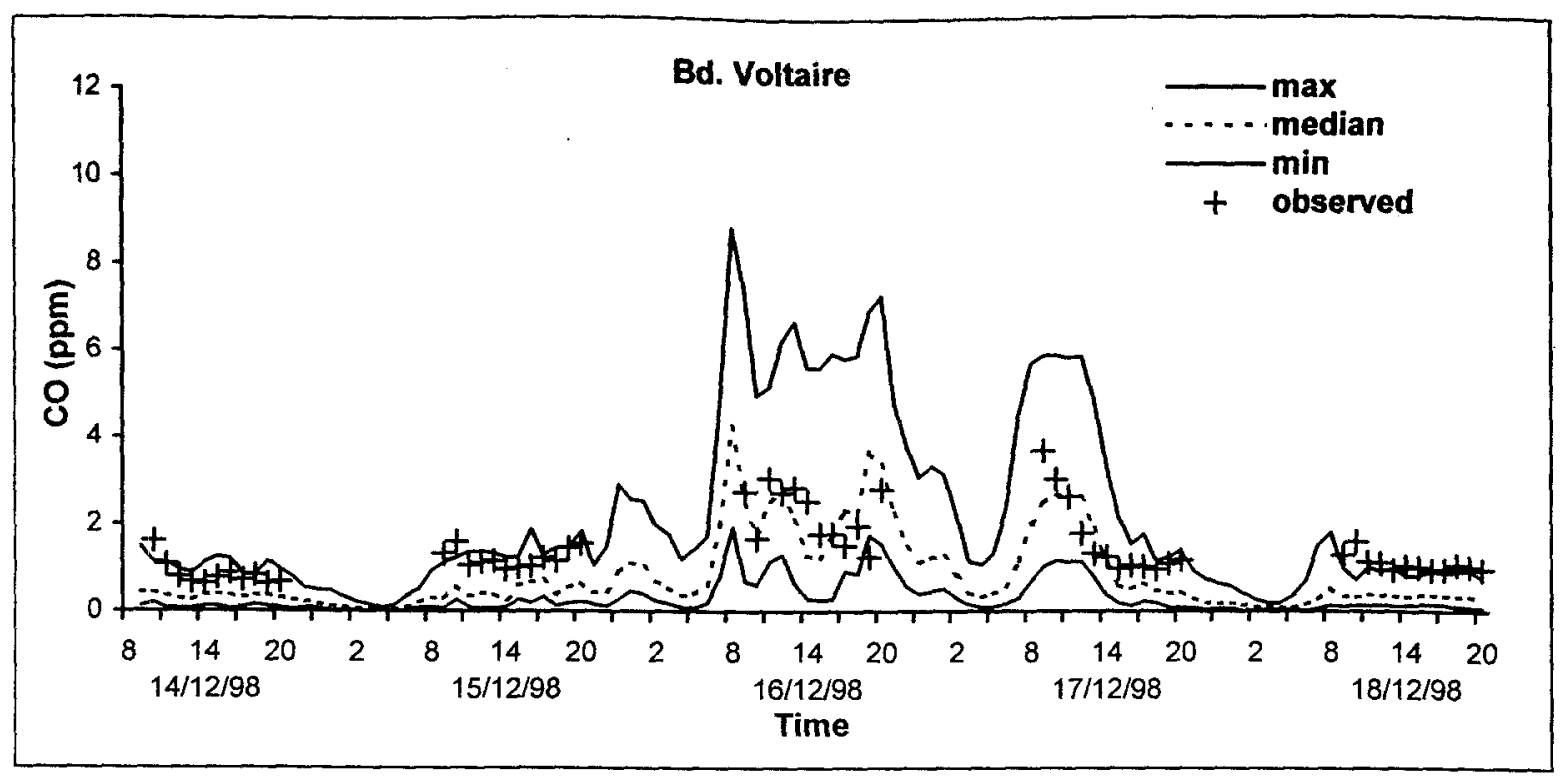

Fig. 4

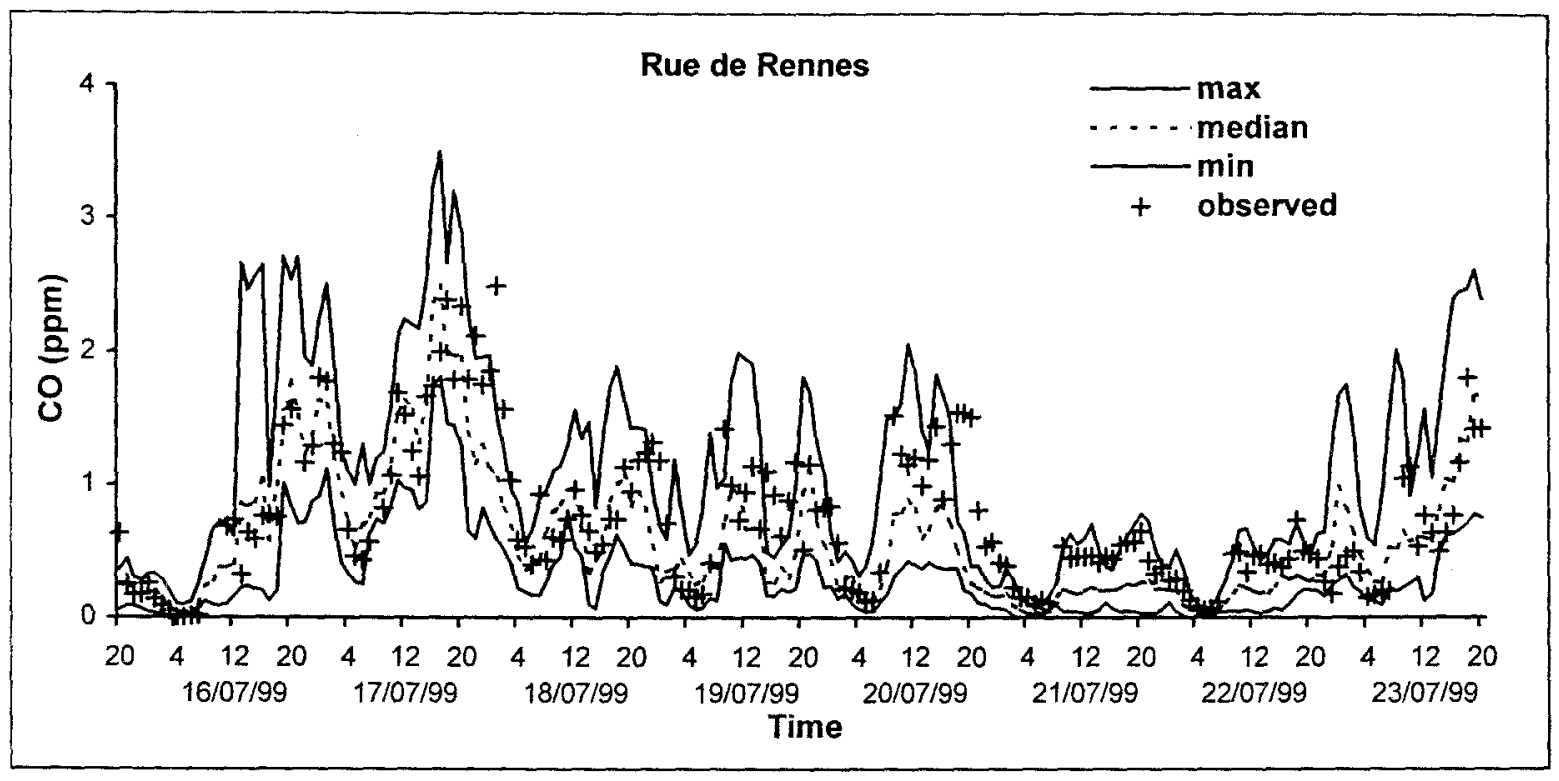

Fig. 5

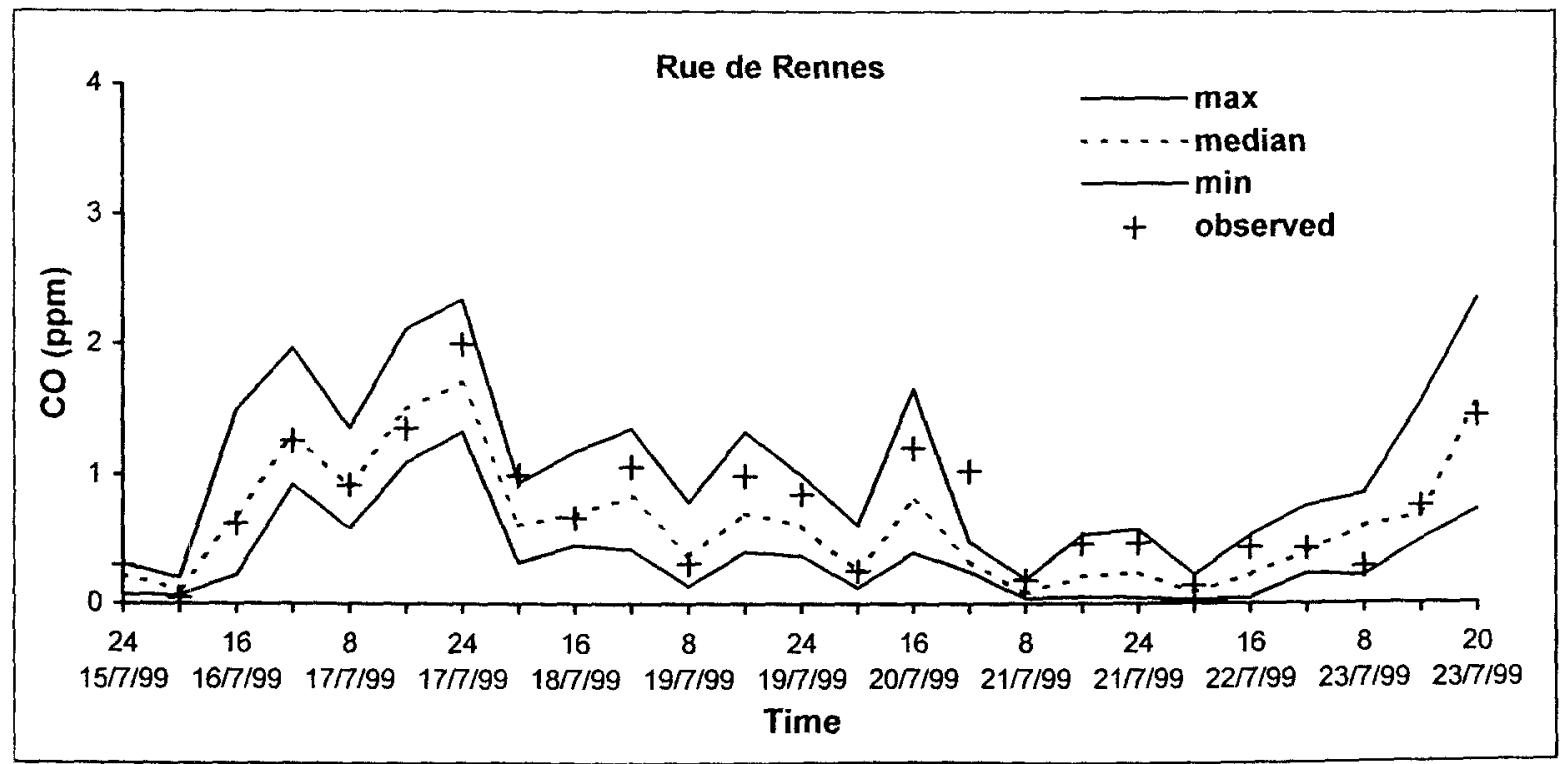

Fig. 6 


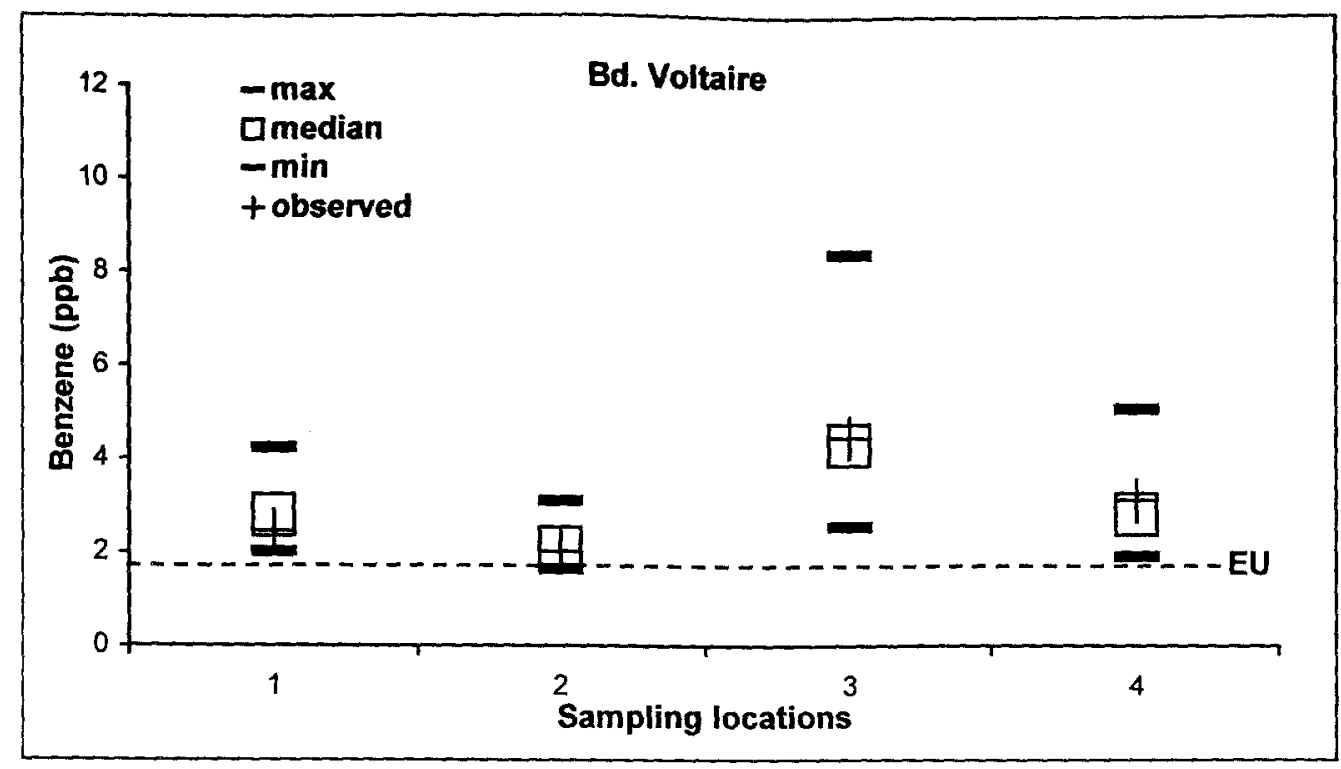

Fig. 7

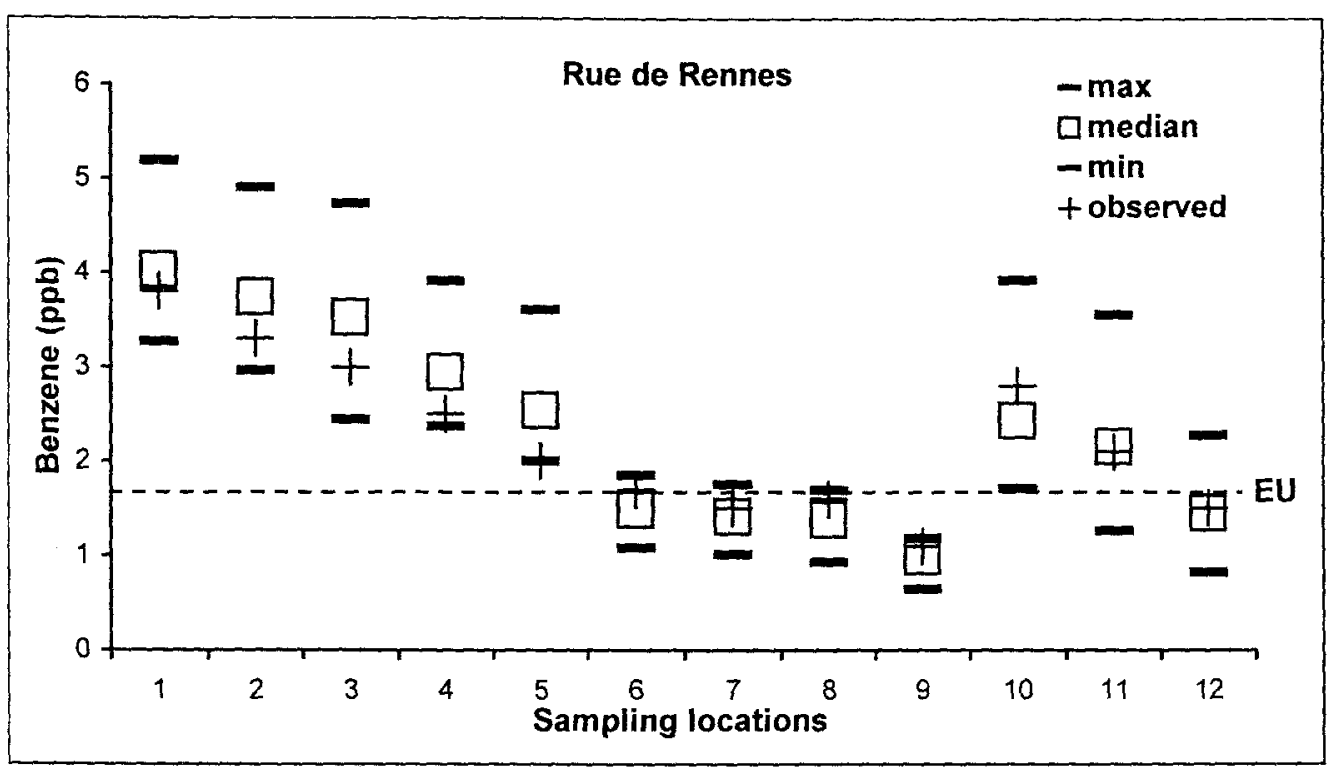

Fig. 8

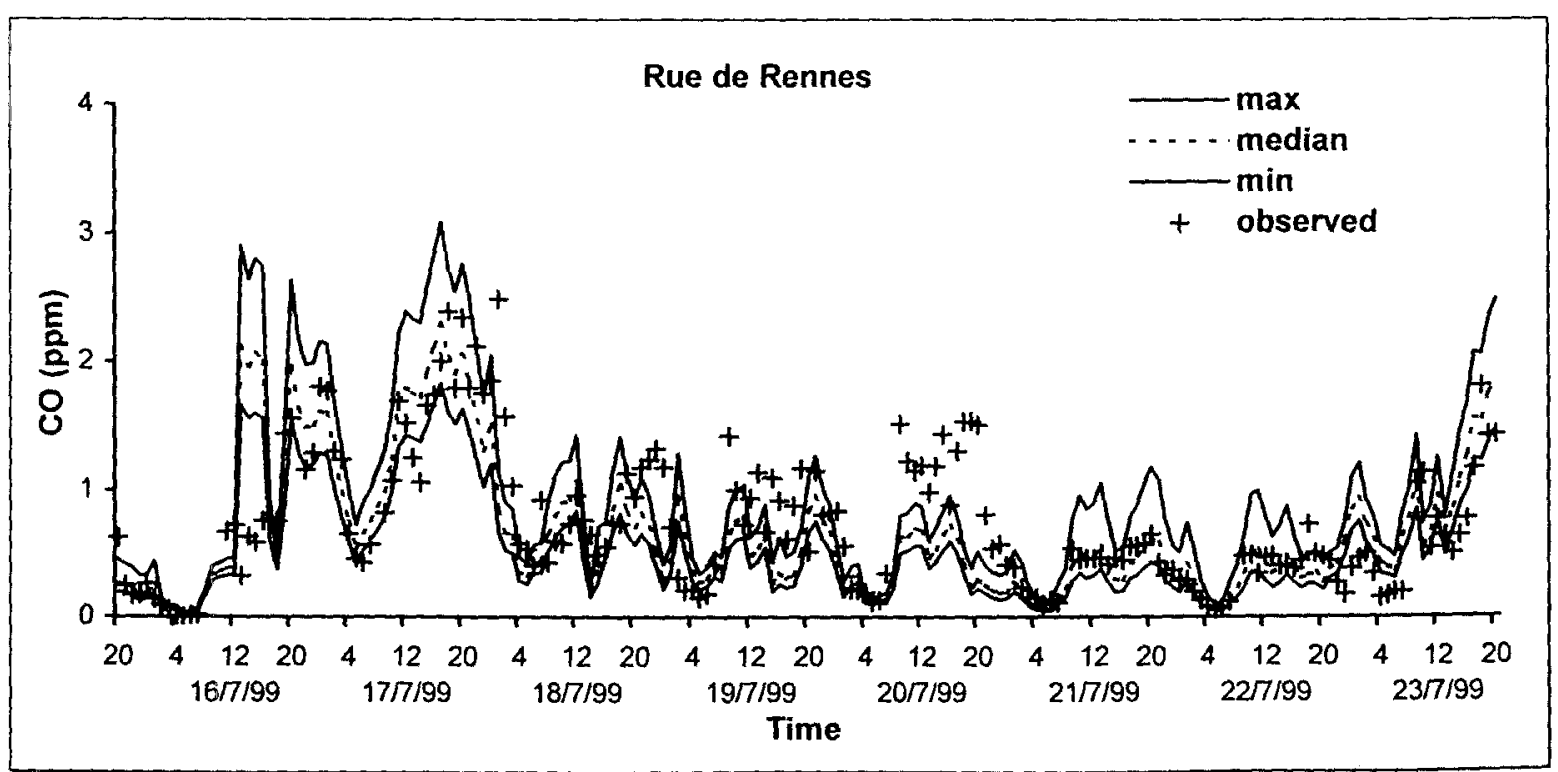

Fig. 9 